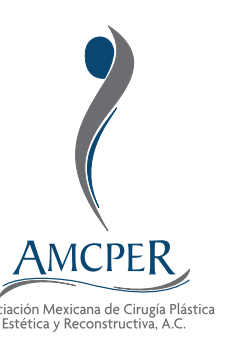

\title{
Sección alta del nervio radial asociada a fractura cerrada de húmero tratada con transferencia nerviosa de nervio mediano a nervio radial sin férula interna
}

\author{
High section of the radial nerve associated with a closed fracture \\ of the humerus treated with a nerve transfer from the median \\ nerve to the radial nerve without an internal splint \\ Israel Salazar-Vizuet,* Mónica Gisela Cobos-Bonilla,* \\ Fernando Franco-Cravioto, ${ }^{\ddagger}$ Fernando Romero-Espinosa ${ }^{\S}$
}

Palabras clave: Parálisis radial alta, transferencia nerviosa, fractura cerrada de húmero.

Keywords: High radial paralysis, nerve transfer, humeral closed fracture.

\section{RESUMEN}

Presentamos el resultado de la transferencia de ramas del nervio mediano para la parálisis del nervio radial después de una fractura humeral. Se realizó transferencia del nervio flexor radial del carpo al nervio interóseo posterior y el nervio del flexor superficial de los dedos al nervio extensor radial del carpo, sin utilizar férula interna, con seguimiento de un año. A los 12 meses postquirúrgicos se recuperó la extensión de la muñeca, de los dedos y del pulgar, con un puntaje de M4. Concluimos que en las fracturas cerradas del húmero Sunderland IV o V del nervio radial se deben realizar transferencias nerviosas.

\section{ABSTRACT}

We present our results in transferring branches of the median nerve for radial nerve palsy following fracture of the humeral shaft. We transferred the flexor carpi radialis to posterior interosseous and flexor digitorum superficialis to extensor carpi radialis brevis without internal splint, with a follow-up of 12 months. Twelve months after surgery the extension of the wrist, fingers and thumb was recovered with a M4 score. We concluded that in closed fractures of the humerus Sunderland IV or V of the radial nerve, nerve transfers should be made.

\section{INTRODUCCIÓN}

L a lesión nerviosa radial asociada con una fractura del húmero es la lesión nerviosa más común e importante en los pacientes con traumatismo del miembro superior. Las fracturas del tercio medio del húmero presentan la mayor incidencia de lesión nerviosa, ya que el nervio se encuentra inmediatamente adyacente al periostio en esta región. ${ }^{1}$ La parálisis del nervio radial se asocia de 6 a $12 \%$ de fracturas en el eje humeral. ${ }^{2}$

Las lesiones del nervio radial pueden resultar en un deterioro motor significativo del brazo y la muñeca, con pérdida de la extensión de la muñeca, de los dedos y de la extensión del pulgar. Las transferencias nerviosas son un nuevo enfoque para el manejo quirúrgico de las lesiones de los nervios radiales grado IV y $\mathrm{V}$ de Sunderland.

Citar como: Salazar-Vizuet I, Cobos-Bonilla MG, Franco-Cravioto F, Romero-Espinosa F. Sección alta del nervio radial asociada a fractura cerrada de húmero tratada con transferencia nerviosa de nervio mediano a nervio radial sin férula interna. Cir Plast. 2020; 30 (3): 149-155. https://dx.doi.org/10.35366/98339 
La asociación entre la lesión del nervio radial y la fractura del húmero fue descrita por los cirujanos de Berkeley Holstein y Lewis, en una publicación de $1983,{ }^{1}$ quienes observaron la parálisis del nervio radial en el establecimiento de fracturas espirales del tercio distal del eje humeral, donde «la punta proximal de esta fractura espiroidal rompe a través de la cortical lateral del húmero muy próximo al punto donde el nervio discurre en estrecho contacto con el hueso y pasa a través del septum intermuscular lateral desde el compartimiento posterior del antebrazo, quedando atrapado el nervio radial en el sitio de la fractura».

Un estudio anatómico publicado recientemente por Carlan y colegas enfatiza que el nervio radial está en riesgo de lesión en estas dos regiones: 1) a lo largo de la parte media posterior del húmero, donde el nervio está en contacto directo con el periostio y, 2) a lo largo del húmero lateral distal, donde el nervio atraviesa el tabique intermuscular lateral. ${ }^{3}$ Las fracturas del tercio medio del húmero presentan la mayor incidencia de lesión nerviosa, debido a que el nervio se encuentra inmediatamente adyacente al periostio en esta región. ${ }^{4}$ La parálisis del nervio radial después de una fractura del eje humeral puede ocurrir después de las fracturas del tercio medio y tercio distal del eje del húmero.
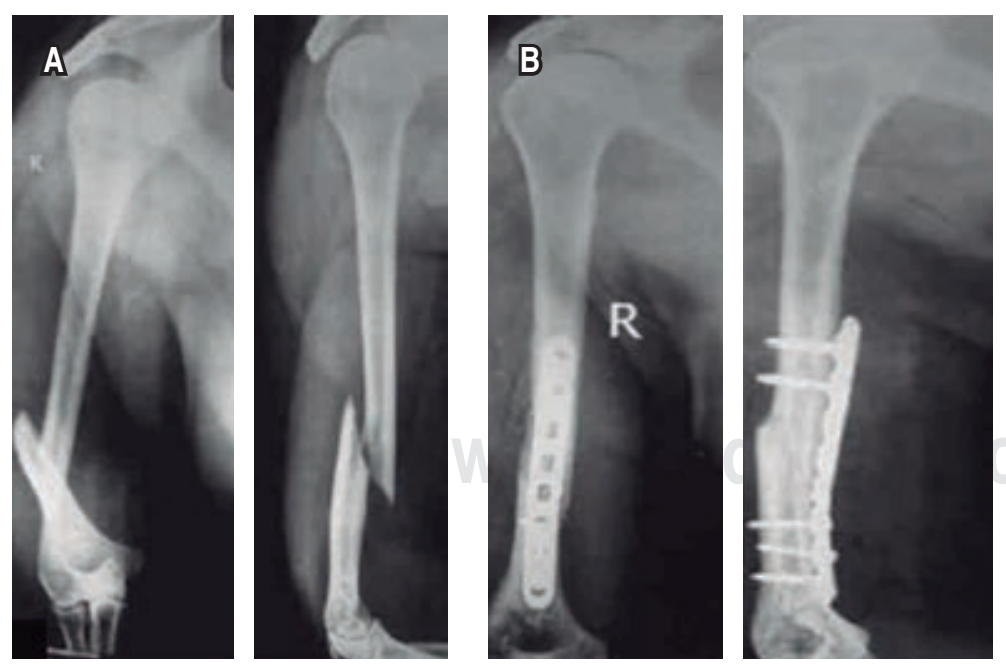

Figura 1: A) Fractura en el tercio distal de húmero derecho. B) Reducción abierta y fijación interna de la fractura mediante la utilización de placas y tornillos.
En la parálisis del nervio radial asociada con la fractura cerrada del húmero, la recuperación espontánea se produce en la mayoría de los pacientes. El músculo braquiorradial y el extensor carpi radialis longus son los primeros músculos en reinervarse. ${ }^{5}$ Los diversos estudios electrodiagnósticos son útiles sólo después de que el proceso de degeneración walleriana se ha establecido, que suele implicar un periodo de tres a seis semanas. En este momento, las lesiones neuropráxicas se habrían recuperado; además, los estudios de electrodiagnóstico no son capaces de distinguir entre un nervio seccionado y un nervio intacto no recuperado y no detectan la recuperación mucho antes que el examen físico. Por lo tanto, cabe destacar que ni los estudios clínicos ni los estudios de electrodiagnóstico ofrecen predictibilidad al tipo de lesión nerviosa en el eje de fractura del húmero.

La recuperación completa suele ocurrir dentro de los seis a los 12 meses después de la lesión; 6 por esta razón, se recomienda el manejo inicial no quirúrgico. Se debe considerar la exploración si no hay evidencia de recuperación clínica seis meses después de la lesión. ${ }^{7}$ La exploración inmediata está indicada en los pacientes con parálisis del nervio radial después de una fractura abierta, y la exploración temprana (dentro de los seis meses) está indicada para los pacientes con lesiones por arma de fuego. $5,6,8,9$

La reparación del nervio puede ser categorizada por el momento de la reparación. Si el nervio se repara en forma aguda en las primeras 72 horas se considera una reparación primaria. La reparación primaria tardía se produce entre 72 horas y una semana. Las reparaciones nerviosas realizadas después de una semana se consideran reparaciones secundarias. Para una transección completa los mejores resultados se producen si la reparación se realiza dentro de las tres semanas de la lesión y un buen pronóstico se puede esperar con las reparaciones realizadas en los primeros seis meses. ${ }^{10} \mathrm{El}$ objetivo principal es conseguir que los axones motores alcancen la placa final del músculo objetivo antes de la fibrosis muscular y la atrofia, que ocurre a los 12 meses; ${ }^{11}$ por tanto, la recuperación funcional es directamente proporcional al número de axones que alcanzan la placa final 

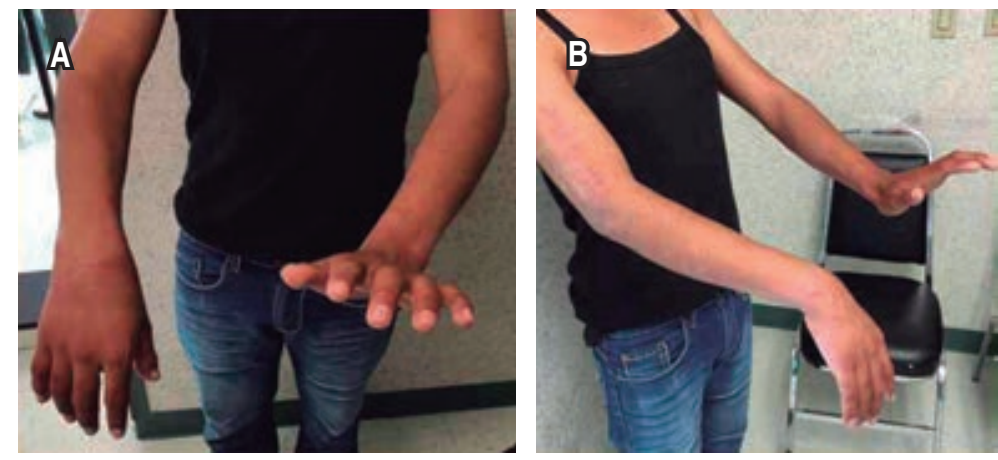

Figura 2: Lesión del nervio radial. A) La lesión al nervio radial da lugar a la pérdida de la extensión de la muñeca y de la extensión del dedo, conocida como muñeca caída. B) Otro ejemplo de caída de muñeca durante un intento de extensión de muñeca y dedo.

objetivo e inversamente proporcional al tiempo de la denervación. El tejido muscular es sensible al tiempo: se someterá a reemplazo graso y fibrosis con el tiempo. La reparación del nervio sensitivo por contraste puede completarse en cualquier momento después de la lesión.

La parálisis del nervio radial se reconstruye típicamente con la reparación directa del nervio, injertos nerviosos o las transferencias tendinosas. Cuando la recuperación espontánea no ha ocurrido o no se espera que ocurra, la función nerviosa radial se ha realizado tradicionalmente con las transferencias tendinosas. Sin embargo, éstas requieren un buen rango de movimiento pasivo y tienen varias desventajas que incluyen disección muscular extensa, biomecánica muscular alterada, inmovilización prolongada, potencial de déficit del donante, ruptura de tendones y adherencias y retorno funcional subnormal. La transferencia nerviosa es una técnica reconstructiva alternativa, que al reinervar la musculatura nativa evita muchos de estos desafíos y ofrece el potencial para un mayor retorno funcional. Específicamente, las transferencias nerviosas para la restauración de la función del nervio radial pueden permitir la extensión del dedo independiente y la extensión simultánea de la muñeca y el dedo.

Los pacientes con lesiones de nervio radial alta que no muestran evidencia de recuperación espontánea, ya sea clínicamente o en EMG (es decir, ausencia de potenciales de unidad motora), o tienen de tres a cuatro meses de la lesión, están indicados para transferencias nerviosas de ramas de nervio mediano a radiales. En otras palabras, son candidatos a realizar una transferencia nerviosa aquellos pacientes que demuestren una lesión radial alta, con lesión axonal grado IV o V de Sunderland.

Este procedimiento está diseñado para restaurar la función de los extensores de la muñeca, de los dedos y del pulgar. La extensión de la muñeca es proporcionada por el extensor carpi radialis longus (ECRL) y el extensor carpi radialis brevis (ECRB). Debido a que ECRB por sí solo proporciona una buena extensión de la muñeca y la rama nerviosa a este músculo proviene del nervio radial propiamente antes de que se convierta en el nervio interóseo posterior (PIN), éstas son las dos ramas que son los objetivos principales para la restauración de la función en lesiones del nervio radial inferior.

Dos transferencias nerviosas ocurren en la transferencia del nervio mediano a radial para la restauración de la extensión de la muñeca y los dedos. Los donantes y los receptores se presentan en los siguientes conjuntos específicos para obtener resultados óptimos con la rehabilitación postoperatoria: 1) flexor radial del carpo a interóseo posterior (PIN) y, 2) flexor digitorum superficialis (FDS) al extensor carpi radialis brevis. La sinergia de esta transferencia facilita la rehabilitación postoperatoria, ya que a través del efecto de la tenodesis la extensión de la muñeca aumenta la flexión del dedo. ${ }^{12,13}$ Se hace intuitivo que el paciente comience a flexionar los dedos (FDS) y después aprenda a extender la muñeca (ECRB), cuando esto sucede la transferencia nerviosa comienza a funcionar. De forma similar, el emparejamiento de la flexión de la muñeca (FCR) con la extensión del dedo inervando, el extensor digitorum communis (EDC) y extensor pollicis longus (EPL) a través del PIN, ayuda a la reeducación por su efecto sinérgico.

Algunos autores sugieren emplear en el mismo procedimiento una férula interna, efectuando una transferencia tendinosa del pronator teres (PT) al extensor carpi radialis brevis (ECRB), para tener una extensión temprana de la muñeca mientras se regenera el nervio, que después mejora una vez que la transferencia de nervios se hace viable. 


\section{CASO CLÍNICO}

Masculino de 18 años de edad, sin antecedentes médicos de importancia. Inició su padecimiento al sufrir caída de un caballo, presenta golpe contuso directo en brazo derecho, con dolor y limitación a la movilización. Acudió 24 horas después con un «huesero», quien aparentemente realizó reducción de fractura, agregándose pérdida de la extensión de la muñeca, pulgar y dedos de la mano derecha. Fue valorado por médico particular quien solicitó radiografía anteroposterior (AP) y oblicua de brazo derecho, la cual mostró presencia de fractura supracondílea de húmero derecho (Figura 1), por lo que fue referido a un Hospital General. Ahí se realizó osteosíntesis de húmero derecho con placa LCP, con hallazgo transoperatorio de sección completa del nervio radial a nivel de la fractura, sin realizar ningún procedimiento de reparación nerviosa.

Fue referido al Servicio de Cirugía Plástica y Reconstructiva dos semanas después del inicio del cuadro. A la exploración física se encontró en la extremidad superior derecha (a la exploración pasiva) mano en flexión. A la exploración activa: incapacidad para la extensión de la muñeca, del pulgar y dedos; las articulaciones metacarpo falángicas en flexión, el pulgar en flexión y aducción (Figura 2) y pérdida de la sensibilidad en territorio del nervio radial.

Se protocolizó para realizar transferencia nerviosa, misma que se realizó bajo anestesia general, sin isquemia, transfiriendo el nervio del flexor carpi radialis a nervio interóseo posterior para la extensión del pulgar y el nervio del flexor común superficial al extensor carpi radialis brevis para lograr la extensión de la muñeca (Figura 3), realizando neurorrafia epineural bajo microscopio con nylon 8-0. No se llevó a cabo férula interna (transferencia tendinosa del pronator teres (PT) al extensor carpi radialis brevis (ECRB)) (Figura 4). En el seguimiento postquirúrgico a los tres, seis y 12 meses se observó mejoría de la extensión de la muñeca y de los dedos M4 y extensión del pulgar M4 (Figura 5), de acuerdo con la Medical Research Council Guidelines.

\section{DISCUSIÓN}

El manejo de la parálisis radial en el contexto de una fractura cerrada de húmero sigue siendo controvertido. El dilema en el manejo de las fracturas cerradas del húmero es no saber si el nervio se encuentra íntegro o seccionado; sin embargo, cuando se cuenta con el diagnóstico de una lesión grado IV o V de Sunderland, el manejo es inminentemente quirúrgico.

Estudios recientes han proporcionado pruebas de que las fracturas se asocian más comúnmente con la neuropraxia del nervio radial, mientras que la neurotmesis es más común en las fracturas abiertas. Existen pocos casos reportados en la literatura de neurotmesis del nervio radial asociados a fracturas cerradas del húmero.

En el estudio realizado por Philipp Leucht, en $2015,{ }^{14}$ hace referencia a dos casos de

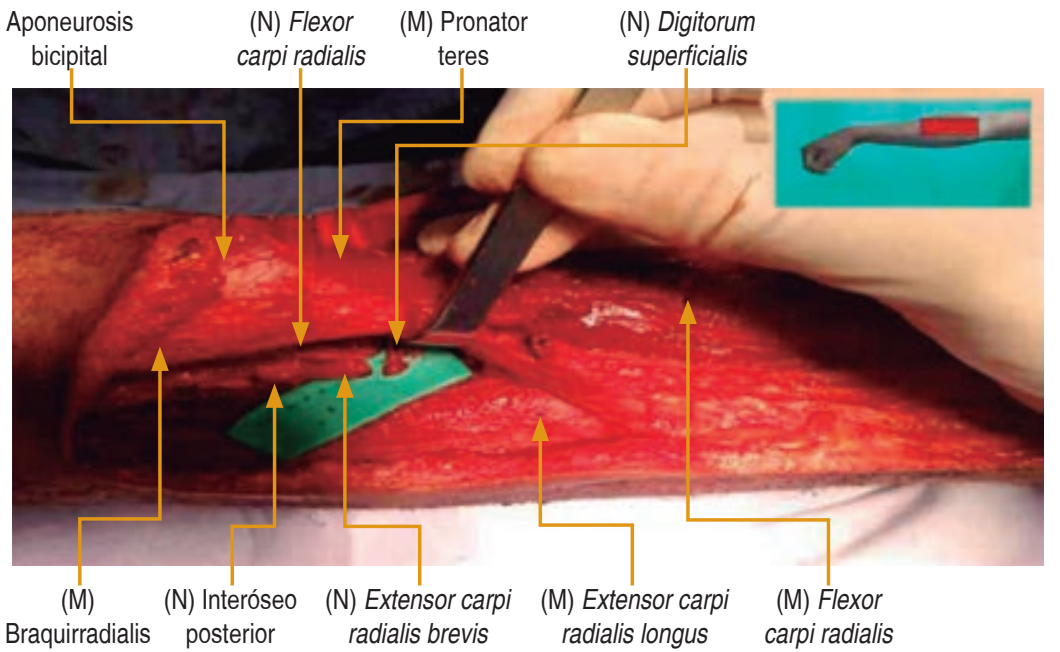

Figura 3:

Se identifican las ramas donadoras del nervio radial y las ramas receptoras del nervio mediano flexor radial del carpo a nervio interóseo posterior, flexor digitorum superficialis al extensor carpi radialis brevis. 


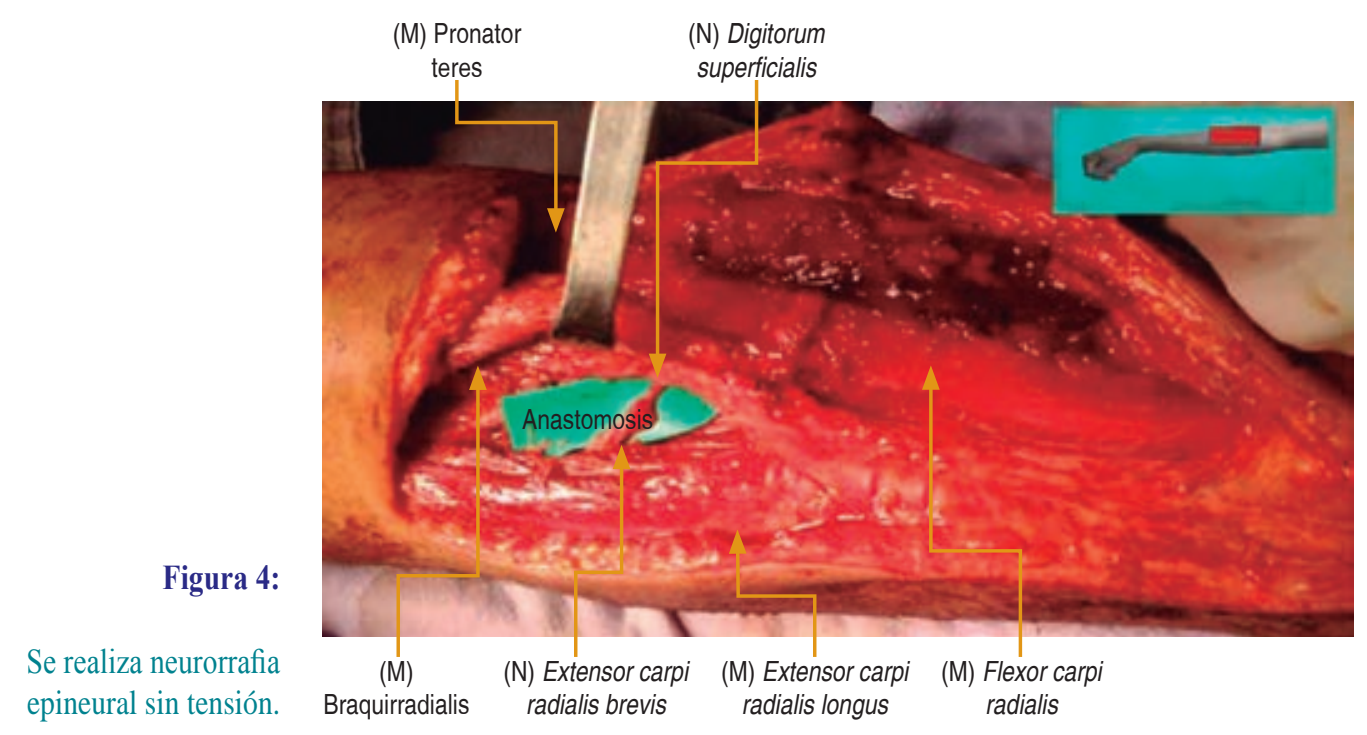

sección completa de nervio radial en fracturas cerradas de húmero, tanto en el tercio distal como en el tercio medio. En ambos casos se solicitó la valoración por el Servicio de Cirugía Plástica y Reconstructiva, en el primer caso tratado a los tres meses se realizó exploración y colocación de injerto nervioso de nervio sural y dos meses después, transferencia nerviosa de la rama motora braquial al nervio mediano. En el seguimiento de un año, el paciente exhibió cierta recuperación del nervio radial con una contracción del braquiorradial y alguna extensión de la muñeca, con eliminación de la gravedad. En el segundo caso se realizó reparación inmediata, interponiendo un aloinjerto de $3 \mathrm{~cm}$ para obtener una reparación sin tensión, sin datos posteriores de recuperación motora. Se observó escasa recuperación motora.

Aunque los injertos nerviosos son un método de restauración del trayecto seccionado, se debe tomar en cuenta la distancia existente entre la sección nerviosa y la placa muscular, puesto que influye en el tiempo de recuperación y este tiempo se traduciría en presencia de fibrosis e infiltración grasa que se presenta en el músculo desnervado a los 12 meses. Las mejores posibilidades de recuperación motora existen cuando el intervalo entre la lesión y la reparación es breve. Aquí es donde aplica también el concepto de las lesiones altas (proximales) contra las bajas (distales), pues si consideramos que la regeneración nerviosa sucede a intervalos de $1 \mathrm{~mm} /$ día desde el sitio proximal de la reparación al sitio del músculo blanco, presentarían intervalos lesión-reparación prolongados. En estos casos, lo que se requiere es dirigir rápidamente una gran cantidad de axones motores a un músculo desnervado y lo más indicado sería la transferencia nerviosa.

Así, las lesiones del nervio proximal situadas en un sitio alejado de la placa final del músculo tienen más distancia para regenerarse, y en última instancia se asocia con peores resultados que en las lesiones más distales, lo que explicaría los pobres resultados de recuperación motora encontrados en el trabajo de Philipp. ${ }^{14}$ Es por ello que abogamos por técnicas de transferencia nerviosa en lesiones proximales siempre que sea posible.

Cuando la recuperación espontánea no ha ocurrido o no se espera que ocurra, se ha restaurado tradicionalmente la función nerviosa radial con las transferencias tendinosas. Sin embargo, éstas requieren un buen rango de movimiento pasivo y tienen varias desventajas, que incluyen disección muscular extensa, biomecánica muscular alterada, inmovilización prolongada, potencial de déficit de donante, ruptura de tendones y adherencias y retorno funcional subnormal. La ventaja de realizar este procedimiento es que se puede realizar en cualquier momento en comparación con 
la transferencia nerviosa, en la que el límite de resultados favorables se ha observado hasta 10 meses después de la lesión. La transferencia nerviosa es una técnica reconstructiva alternativa que al reinervar la musculatura nativa evita muchos de estos desafíos y ofrece el potencial para un mayor retorno funcional.

En el caso que presentamos, debemos tomar en cuenta que se trata de un paciente joven, económicamente en edad productiva, con una lesión motora importante en la mano derecha (mano dominante), que interfiere con la capacidad para realizar actividades diarias y su trabajo.

El paciente fue referido en un periodo superior a los siete días posteriores desde el accidente a su admisión en el Servicio de Cirugía Plástica y Reconstructiva con clínica de parálisis radial alta y con referencia de envío que menciona sección completa de nervio radial a nivel de la fractura en el brazo derecho (mano dominante). Se realizó transferencia nerviosa del nervio mediano a nervio radial, sin elaborar férula interna, que consiste en realizar una transferencia tendinosa del pronator teres (PT) al extensor carpi radialis brevis (ECRB) durante el mismo procedimiento.
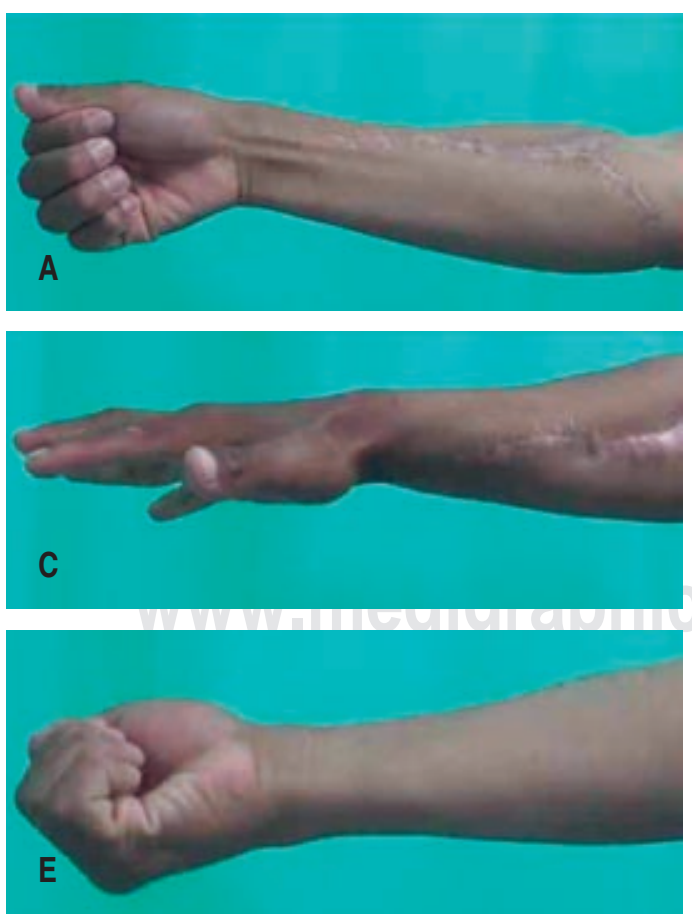

En su seguimiento en la consulta externa se inició rehabilitación a los 14 días de la cirugía y se observó una recuperación parcial a los tres meses con presencia de extensión de la muñeca y de los dedos M2; mientras que del pulgar M2 al año de seguimiento, con extensión de la muñeca y de los dedos M4, mientras que del pulgar M3, de acuerdo al sistema del Medical Research Council.

\section{CONCLUSIONES}

En las fracturas cerradas de tercio distal del húmero con evidencia de sección completa de nervio radial alta Sunderland IV o V (neurotmesis), se deben realizar transferencias nerviosas lo más pronto posible para obtener mejores resultados, ya que la distancia existente entre la sección nerviosa y la placa muscular influye en el tiempo de recuperación, y el tiempo que tardaría la recuperación se traduciría en presencia de fibrosis, cicatrices e infiltración grasa del músculo que se presenta en el músculo desnervado a los 12 meses. En estos casos lo que se requiere es dirigir rápidamente una gran cantidad de axones motores a un músculo desnervado, lo que se logra con la transferencia nerviosa.
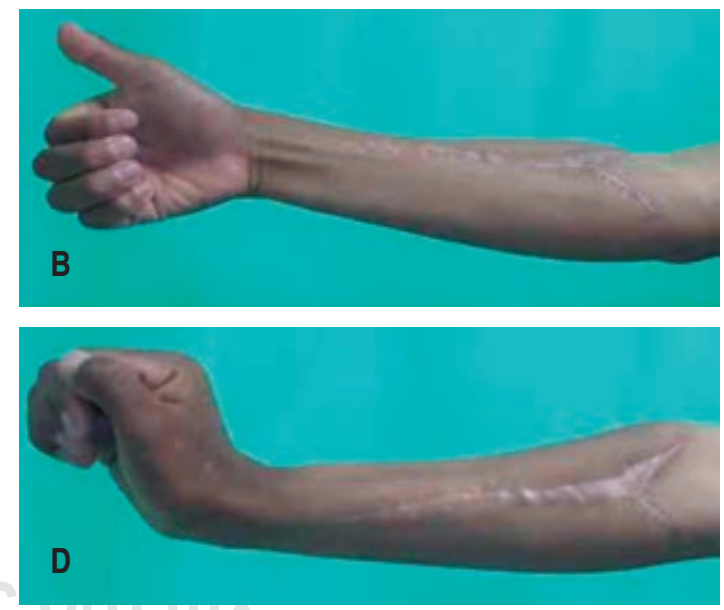

Figura 5:

Evaluación de la actividad motora al año de la realización de la transferencia nerviosa. A y B) Oposición y extensión del pulgar. C-E) Extensión de los dedos, extensión de la muñeca, presencia de flexión y pronación, respectivamente. 


\section{REFERENCIAS}

1. Holstein A, Lewis GB. Fractures of the humerus with radial-nerve paralysis. J Bone Joint Surg 1963; 45A: 1382-1388.

2. Ekholm R, Adami J, Tidermark J, Hansson K, Törnkvist $\mathrm{H}$, Ponzer S. Fractures of the shaft of the humerus, an epidemiological study of 401 fractures. J Bone Joint Surg 2006; 88B: 1469-1473.

3. Carlan D, Pratt J, Patterson JMM, Weiland AJ, Boyer MI, Gelberman RH. The radial nerve in the brachium: an anatomic study in human cadavers. J Hand Surg 2007; 32A: 1177-1182.

4. Ashfaq Hasan S, Rauls RB, Cordell CL, Bailey MS, Nguyen T. "Zone of vulnerability" for radial nerve injury: anatomic study. J Surg Orthop Adv 2014; 23:105-110.

5. DeFranco MJ, Lawton JN. Radial nerve injuries associated with humeral fractures. J Hand Surg 2006; 31A: 655-663.

6. Shao YC, Harwood P, Grotz MRW, Limb D, Giannoudis PV. Radial nerve palsy associated with fractures of the shaft of the humerus. J Bone Joint Surg 2005; 87B: 1647-1652.

7. Packer JW, Foster RR, Garcia A, Grantham SA. The humeral fracture with radial nerve palsy: is exploration warranted? Clin Orthop 1972; 88: 34-38.

8. Kettelkamp DB, Alexander H. Clinical review of radial nerve injury. J Trauma 1967; 7: 424-432.

9. Pollock FH, Drake D, Bovill EG, Day L, Trafton PG. Treatment of radial neuropathy associated with fractures of the humerus. J Bone Joint Surg 1981; 63A: 239-243.
10. Novak CB, Mackinnon SE. Distal anterior interosseous nerve transfer to the deep motor branch of the ulnar nerve for reconstruction of high ulnar nerve injuries. J Reconstr Microsurg 2002; 18: 459-464.

11. Lowe JB, Tung TR, Mackinnon SE. New surgical option for radial nerve paralysis. Plast Reconstr Surg 2002; 110: 836-843.

12. Mackinnon SE, Roque B, Tung TH. Median to radial nerve transfer for treatment of radial nerve palsy: case report. J Neurosurg 2007; 107: 666-671.

13. Ray WZ, Mackinnon SE. Clinical outcomes following median to radial nerve transfers. J Hand Surg Am 2011; 36: 201-208.

14. Leucht P, Ryu JH, Bellino MJ. Radial nerve transection associated with closed humeral shaft fractures: a report of two cases and review of the literature. J Shoulder Elbow Surg 2015; 24 (4): e96-100.

\section{Correspondencia:}

\section{Dr. Israel Salazar Vizuet}

Nicolás San Juan S/N Esq. Ganadería y Parque, Rancho Cuauhtémoc,

Col. Ex Hacienda Magdalena, 50010, Municipio Toluca, Estado de México.

E-mail: israelsv23@gmail.com

Conflicto de intereses: Los autores declaran no tener conflicto de intereses. 